\title{
Kernos
}

Revue internationale et pluridisciplinaire de religion grecque antique

$27 \mid 2014$

Varia

\section{Un aition per due feste}

Una proposta di lettura dei Daidala in Pausania IX, 2, 7-3, 8

\section{Fiorella La Guardia}

\section{Q OpenEdition \\ 1 Journals}

\section{Edizione digitale}

URL: http://journals.openedition.org/kernos/2279

DOI: $10.4000 /$ kernos.2279

ISSN: 2034-7871

\section{Editore}

Centre international d'étude de la religion grecque antique

\section{Edizione cartacea}

Data di pubblicazione: 1 novembre 2014

Paginazione: 177-205

ISBN: 978-2-87562-055-2

ISSN: 0776-3824

Notizia bibliografica digitale

Fiorella La Guardia, «Un aition per due feste », Kernos [En ligne], 27 | 2014, mis en ligne le 01 octobre 2016, consulté le 01 mai 2019. URL : http://journals.openedition.org/kernos/2279; DOI : 10.4000/ kernos. 2279

Questo documento è stato generato automaticamente il 1 maggio 2019.

Kernos 


\title{
Un aition per due feste
}

Una proposta di lettura dei Daidala in Pausania IX, 2, 7-3, 8 *

\author{
Fiorella La Guardia
}

“... le duc d'Auge se pointa sur le sommet du

donjon de son château pour y considérer, un tantinet soit peu, la situation historique. Elle était plutôt floue. Des restes du passé traînaient encore

çà et là, en vrac"

R. Queneau, Les fleurs bleues.

Per Alessia

1 Oggetto di questa indagine sono le due feste dei Daidala (Daidala Mikra e Daidala Megala) come testimoniate nel libro di Pausania sulla Beozia (IX) e l'aitionche il Periegeta pone alla loro base. Riferimenti all'istituzione della festa, e all'aition ad essa relativo, si trovano, con ovvie varianti, anche in una perduta opera plutarchea dedicata proprio ai Daidala (Пвpì

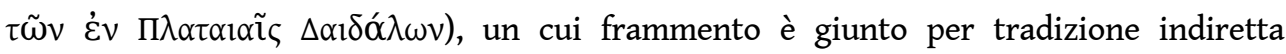
attraverso Eusebio ${ }^{1}$.

2 La scelta di Pausania per la presente ricerca è motivata dalla ricchezza di dettagli che il Periegeta fornisce sugli aspetti rituali, sui quali si intende qui concentrarsi ${ }^{2}$. Obiettivi dell'indagine saranno : 1 . l'analisi dell'aition e del modo in cui esso si lega agli elementi del rito/dei riti (relazione tra riti matrimonialie olocausto); 2.l'analisi della festa e l'individuazione dei caratteri differenziali tra le due forme testimoniate nel testo ; 3 . il tentativo di comprensione dell'oggetto $\delta \alpha i ́ \delta \alpha \lambda \alpha$ e del suo statuto all'interno del rito (simbolo di cosa ?) ; 4. la messa in rilievo del carattere stratificato del testo, che presenta elementi che si devono supporre diversi per origine e diacronia.

Quanto al testo di Plutarco, esso costituirà esclusivamente termine di confronto occasionale per quegli aspetti per i quali sarà utile ingadare gli elementi testimoniati da una diversa tradizione, che pure paiono in qualche modo collegati a ciò che troviamo in Pausania. Vediamo dunque, anzitutto, il dettato del testo : 
$[2,7]$ I Plateesi hanno un tempio di Era, degno di nota per grandezza e per le statue che lo adornano. All'entrata si trova una statua di Rea, che porta a Crono il sasso avvolto in fasce, come se fosse il figlio che ha partorito; poi una statua di Era che chiamano Teleia, stante e di notevole grandezza; entrambe di marmo pentelico, sono opera di Prassitele. Nello stesso tempio si trova anche un'altra statua di Era, seduta, che ha realizzato Callimaco ; chiamano la dea Nympheuomene per la seguente ragione. $[3,1]$ Dicono che Era, adirata con Zeus per una qualche ragione si fosse ritirata in Eubea, e raccontano che Zeus, poichè non riusciva a farle cambiare idea, si fosse recato da Citerone che era allora il signore di Platea; per sapienza infatti Citerone non era secondo a nessuno. Questi dunque consiglia a Zeus di realizzare una statua di legno e di trasportarla coperta da un velo su un carro trainato da una coppia buoi, dicendo che conduceva in sposa Platea, figlia di Asopo. [3, 2] E il dio faceva secondo il consiglio di Citerone; ma Era, venuta a sapere subito la cosa, subito giunse. Come si avvicinò al carro e squarciò il drappo della statua, si accorse dell'inganno avendo trovato una statua di legno al posto di una sposa e fece pace con Zeus. In ragione di questa riconciliazione celebrano la festa dei Daidala, poichè gli antichi chiamavano daidala le statue di legno ; e le chiamavano così - a me pare - ancor prima che nascesse in Atene Dedalo, figlio di Palamaone, e che a lui il nome sia stato posto in seguito come soprannome derivato dai daidala, e non sin dalla nascita. [3, 3] I Plateesi dunque celebrano la festa dei Daidala ogni sei anni, a quanto diceva la guida locale, secondo un computo corretto, tuttavia, in un intervallo di tempo più breve e non così lungo; ma volendo calcolare con la massima esattezza l'intervallo di tempo intercorrente tra una celebrazione dei Daidala e l'altra, non ne fummo in grado. Celebrano la festa in questo modo. [3,4] Non lontano da Alalcomene c'è un bosco di querce : qui i fusti delle querce sono i più grandi della Beozia. Giunti a questo bosco, i Plateesi vi dispongono pezzi di carne cotta. Di tutti gli altri uccelli essi non si danno minimamente cura, ma ai corvi - questi infatti si avvicinano ad essi - pongono invece molta attenzione. Scrutano su quale degli alberi si posi quello tra i corvi che ha afferrato la carne. E avendo tagliato l'albero sul quale il corvo si sia posato, da esso realizzano il daidalon. Infatti chiamano daidalon proprio la statua lignea ( celebrano per conto proprio e la chiamano Daidala Mikra; la festa dei Daidala Megala la celebrano invece tutti i Beoti insieme, ogni sessant'anni; dicono infatti che per altrettanto tempo avevano sospeso la festa, nel periodo in cui i Plateesi erano in esilio. Essi hanno pronte quattordici statue lignee, realizzate nel corso di ogni celebrazione dei Daidala Mikra. [3, 6] Queste statue se le spartiscono a sorte Plateesi, Coronei, Tespiesi, Tanagrei, Cheronei, Orcomenii, Lebadei e Tebani ; anche questi infatti chiesero di riconciliarsi $(\delta 1 \alpha \lambda \lambda \alpha \gamma \tilde{\eta} v \alpha \mathrm{l})$ con i Plateesi, di partecipare alla

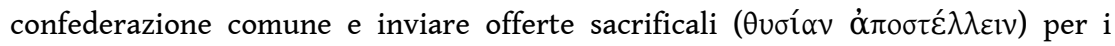
Daidala, quando Cassandro figlio di Antipatro rifondò Tebe. [3, 7] Avendo adornato

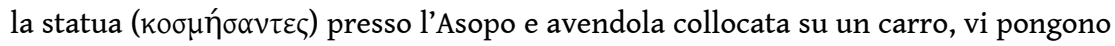
accanto una donna che faccia da nympheutria; ed essi di nuovo fanno un sorteggio per stabilire l'ordine con cui sfileranno in processione. Di qui guidano poi i carri dal fiume fin sulla cima del Citerone. Ed è stato apprestato per essi sulla cima del monte

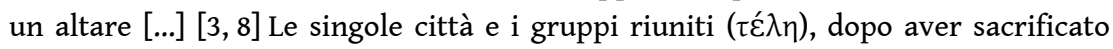
ciascuno una vacca ad Era e un toro a Zeus, bruciano sull'altare le vittime sacrificali, piene di vino e essenze e, assieme ad esse, i daidala, mentre i privati cittadini sacrificano vittime quante ne vogliano, se ricchi ; nel caso invece di coloro che non abbiano altrettante disponibilità, è uso che sacrifichino capi di bestiame più piccoli, ma bruciano tutte le vittime al medesimo modo [...]; e questo rogo è il più grande che io abbia mai visto levarsi ed essere visibile da molto lontano.

4 Fin qui il racconto di Pausania.

5 Avverto in premessa che non mi occuperò di alcune questioni, che pure sono problematiche o degne di interesse, ma che non investono l'interpretazione del rito in quanto tale, e cioè in particolare : 1. l'incongruenza tra il numero delle statuette (14) e la 
periodicità dei Daidala Megala (60 anni) rispetto alla indicata periodicità dei Daidala Mikra (cui viene attribuito un ritmo eptaeterico) ${ }^{3} ; 2$. la congruenza tra l'elenco di città e tele partecipanti ai Daidala Megala e l'effettivo assetto territoriale della Beozia; 3. l'incongruenza tra i periodi di esilio dei Plateesi come testimoniati dalle fonti storiche e la durata di quello cui fa riferimento Pausania ${ }^{4}$ (il fatto cioè che tra i periodi di esilio dei Plateesi testimoniati storicamente nessuno sia durato $60 \mathrm{anni)}$; 4. la relazione tra daidala e Daidalos come personaggio mitico (IX, 3, 2) e i legami che l'oggetto intrattiene con gli

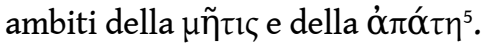

6 La ricerca si è mossa su un doppio versante, quello delle fonti letterarie e quello dell'evidenza materiale. Per quanto riguarda le prime, si sono presi in considerazione, da una parte, passi relativi al culto di Era (con particolare riferimento a Platea e la Beozia, ove possibile $o$, in alternativa, ai principali luoghi di venerazione della dea) e agli aspetti che appaiono ad esso associati ; dall'altra, fonti che offrano testimonianza di operazioni rituali aventi ad oggetto dei simulacri (con particolare riferimento naturalmente a contesti nei quali compaia Era) o che avessero a che fare con l'ambito matrimoniale.

Quanto all'evidenza materiale, ciò che si è preso in considerazione consiste essenzialmente nelle testimonianze relative al culto di Era in Beozia - con particolare riferimento alla cronologia del culto stesso e agli epiteti testimoniati - all'Heraion di Platea, all'eventuale associazione di statuette col culto di Era.

Concludo queste premesse sottolineando come, nella storia degli studi dedicati al testo, la critica si sia sostanzialmente distinta in due gruppi ${ }^{6}$ : coloro che hanno visto come nodo centrale quello che, in modo non del tutto corretto a mio avviso, viene descritto come

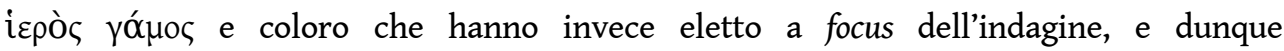
dell'interpretazione, l'olocausto - gli uni e gli altri facendo quasi sempre rimarcare l'impossibile conciliazione tra i due elementi. Cercheremo di vedere, attraverso l'analisi, in che rapporto essi stiano e se ci sia conciliazione possibile.

\section{La logica del testo}

9 Pausania introduce l'aition che sta alla base del lungo excursus sui Daidala a spiegazione

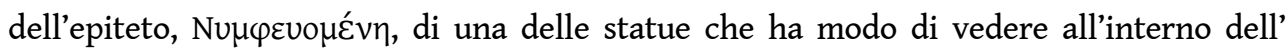
Heraion di Platea : ci dice infatti di aver visto una statua di Rea ed una di Era Teleia, opera di Prassitele, e una ulteriore statua di Era, Nympheuomene, ("che viene condotta in sposa", è questo il senso che si deve attribuire al termine), realizzata da Callimaco.

Uno sguardo attento alla struttura del testo fa emergere come, di fatto, l'aitionsi leghi in modo diretto solo alla seconda delle due feste narrate, mentre la descrizione della prima appare una sorta di inserto volto a illustrare la complessa procedura ${ }^{7}$ con la quale viene selezionato il materiale per realizzare il daidalon, senza che nessuna indicazione sia fornita sull'impiego che esso riceve una volta realizzato.Particolarmente indicative di tale logica costruttiva del testo appaiono tanto la non perfetta conseguenzialità tra

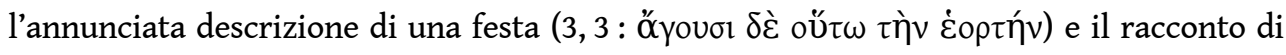

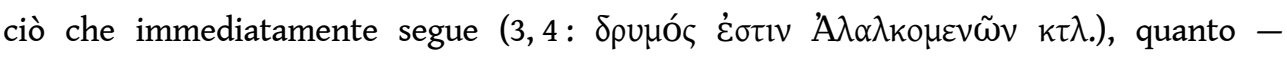

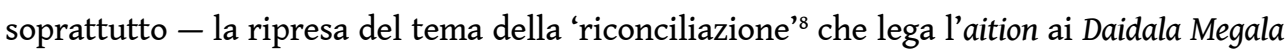
appunto, mentre non mostra alcuna connessione con i Daidala Mikra. Tali discontinuità mostrano come l'interpretazione dei dati di cui disponiamo sia resa particolarmente complessa dalla evidente stratificazione, all'interno del testo, di elementi eterogenei, dei 
quali non è sempre possibile rintracciare la matrice, stratificazione che deve essere ricondotta da una parte all'autore che ce ne conserva testimonianza, alle distorsioni generate dalla lunga diacronia che lo separa dalle cose narrate, dall'altra - soprattutto a ciò che Angelos Chaniotis imputa alla dinamica dei rituali, alla dialettica esistente cioè "between ritual actions, which have to be performed in a particular way, and the continually changing community of performers, participants, and receptors or spectators". Quest'ultimo aspetto risalta con particolare evidenza, come si avrà modo di notare, nelle incongruenze esistenti tra elementi (i più numerosi) che possono essere ricondotti all'universo simbolico del matrimonio e altri, fuori serie, che richiedono di essere isolati e problematizzati e che inducono a presumere una avvenuta rifunzionalizzazione degli oggetti che costituiscono il focus del rito e dell'universo simbolico che ad essi si lega.

11 Proprio l'epiteto dal quale l'excursus prende avvio e l'altro, non direttamente legato all' aition(Teleia),devono essere tenuti a mente e, credo, posti in valore - come cercherò di mostrare - alla ricerca di possibili elementi di organicità del quadro rituale che, nella forma riflessa nel testo, pone problemi rispetto ai legami esistenti tra alcune delle sue componenti.

\section{Antichità del culto di Era in Beozia}

Dalle fonti archeologiche sappiamo che il culto di Era in Beozia è databile già dall'età micenea (essa compare nelle tavolette tebane tra le divinità destinatarie di vari materiali, come p. es. in TH of 28), e che la dea era qui venerata con una serie di epiteti, alcuni dei quali - come Kithaironia e Teleia - sono di immediato interesse rispetto al nostro testo. Una tavoletta in particolare (TH Av 104) contiene elementi di rilievo ${ }^{10}$ : troviamo in essa la menzione di gruppi di individui, designati come si-to-ko[-wo $0^{11}$, che secondo le interpretazioni correnti sarebbero dei ministri del sacro legati a una particolare divinità ${ }^{12}$ , o piuttosto degli addetti alle risorse in grano e cereali dei santuari ${ }^{13}$, posti in relazione con delle località espresse con l'allativo (la forma che traduce nel suffisso l'idea del moto a luogo) ; tra queste località, Te-re-ja-de (l. 2) ${ }^{14}$. Avremmo ciò̀ la testimonianza di gruppi di individui inviati da Teleia o presso il santuario di Teleia ${ }^{15}$.

Secondo Schachter (che ai Culti della Beozia ha dedicato una monografia ${ }^{16}$ e che a più riprese si è occupato delle specificità epicoriche di alcune divinità per quella regione ${ }^{17}$ ), $\mathrm{i}$ vari dati relativi all'epiteto Teleia dovrebbero far riferimento alla strutturazione della Beozia per tele, per distretti cioè, e solo in piena età storica l'interpretazione di Teleia come "Era adulta" avrebbe condotto a stabilire una sostanziale equivalenza tra Teleia e Nympheuomene ${ }^{18}$. Tali osservazioni mi paiono difficilmente condivisibili, per più ragioni : anzitutto l'epiteto Teleia è associato ad Era anche in contesti che hanno strutturazione assai differente da quella dell'ethnos beotico (non per tele cioè); d'altra parte, il legame dell'epiteto con telos e il fatto che la dea qualificata come Teleia sia costantemente presentata nelle fonti scritte come associata al matrimonio ${ }^{19}$ lasciano pochi dubbi $-\mathrm{mi}$ pare - sulla pregnanza religiosa del termine. A ciò si aggiunge che - come sarà chiaro dallo svolgimento dell'analisi - i due termini (Nympheuomene e Teleia) non possono essere considerati equivalenti. riguardo all'antichità del culto (dal materiale di età micenea la dea appare come poliouchos 
di Plate ${ }^{20}$ ) e la testimonianza congiunta di evidenza archeologica e fonti letterarie ci fa certi del rilievo che l'Heraion plateese, e dunque il culto di Era, dovevano avere già in epoca ben anteriore a quella testimoniata da Pausania ${ }^{21}$.

\section{Daidala Mikra e i caratteri dell'idolo arcaico}

15 In che modo, dunque, Era e il suo culto sono legati ai Daidala, a una festa cioè, il cui nome denuncia con chiarezza come focus del rito dovessero essere le statuette? ${ }^{22}$ L'elemento connettivo è rappresentato, a livello dell'aition, dalla falsa sposa (Platea) che, sotto forma di statuetta (di daidalon ${ }^{23}$ ), Zeus realizza su consiglio di Citerone per provare a sciogliere l'ira della dea e la sua decisione di isolamento in Eubea. Dal felice esito della vicenda, conclusa dalla riconciliazione tra i due sposi divini, nasce l'istituzione, da parte dei Plateesi, dei Daidala come festa, poichè $\delta \alpha i ́ \delta \alpha \lambda \alpha$ - chiarisce Pausania - è il nome con il quale gli antichi chiamavano gli łó $\alpha v \alpha$, gli antichi simulacri di legno (IX, 3, 2).

L'analisi etimologica non fornisce elementi utili alla individuazione di caratteri differenziali del daidalon rispetto allo xoanon o alle altre forme della rappresentazione figurata. Della famiglia cui il termine appartiene, Chantraine nota semplicemente che si tratta di parole poetiche, che sono antiche e le cui forme nominali sono più importanti di quelle verbali ${ }^{24}$. Maggiori informazioni si ricavano dalla analisi sistematica che Françoise Frontisi-Ducroux ha dedicato a "l'ensemble des termes qui composent la famille lexicale de l'anthroponyme Daidalos" ${ }^{25}$. Gli oggetti cui tali termini si riferiscono ${ }^{26}$ appartengono essenzialmente a tre categorie : oggetti in metallo (di gran lunga i più numerosi), oggetti in legno, tessuti, e i contesti nei quali essi compaiono fanno chiaro come la loro essenza non sia confinata a una realtà di ordine puramente tecnico, ma rimandi a un sistema più vasto, il sistema costituito da tutti quegli oggetti che Louis Gernet raggruppa entro la classe degli agalmata ${ }^{27}$.

Istituita dunque l'equivalenza daidalon-xoanon, e rovesciato il rapporto, evidentemente diffuso nella tradizione, tra Daidalos e daidalon, ciò che il testo ci presenta, e che viene indicato da Pausania come contenuto della festa plateese, consiste di fatto nella descrizione assai dettagliata della procedura, dai marcati caratteri rituali, attraverso la quale viene operata la selezione dell'albero con il quale sarà realizzato il daidalon. Il contenuto di questa sezione (IX, 3,4) presenta elementi che sono comuni nel mito: l'affidamento ad un animale di una sorta di compito oracolare ${ }^{28}$, il legame fra una specifica specie arborea e una singola divinità ${ }^{29}$, i caratteri di bellezza e perfezione che il materiale scelto per realizzare un agalma destinato alla divinità deve avere. Sono qui significativamente condensati $i$ tratti che Jean-Pierre Vernant descrive come propri dell'idolo arcaico. Nel dattiloscritto inedito che conserva parte della incompiuta Thèse sulla Figura degli dei ${ }^{30}$, Vernant osserva, a proposito dello xoanon, che "en lui c'est la matière [...] qui a valeur symbolique : le bois, tel bois, tel arbre, en rapport direct avec la divinité" ${ }^{1}$. L'importanza del passaggio in questione risiede nella possibilità di affermare con buona certezza, grazie alle possibili 'associazioni' con altri contesti mitici/rituali, che gli elementi in esso conservati rinviano ad uno stadio di civiltà e dunque a dati di mentalità assai più antichi dell'autore che ce ne conserva testimonianza. La sezione di testo cui abbiamo fatto riferimento è chiusa da una nuova affermazione della equivalenza daidalon $=x$ xonon $^{32}$, ma nessuna indicazione è fornita sulla destinazione del simulacro, che rimane dato problematico in quanto è difficile immaginare che esso, una volta realizzato, non avesse alcun impiego rituale; tutto quanto possiamo dedurre, sulla base delle 
indicazioni che il nostro autore ci fornisce, è che i simulacri via via realizzati nel corso dei Daidala Mikra fossero custoditi per essere poi utilizzati in occasione della festa maggiore ${ }^{33}$. Nuove informazioni ci vengono fornite infatti solo a proposito dei Daidala Megala, la festa panbeotica la cui descrizione segue immediatamente : i simulacri vengono condotti sulle rive dell'Asopo, dove saranno presumibilmente lavati ${ }^{34}$, e poi collocati su carri (ciascuno con una nympheutria accanto), che formeranno la pompe che deve condurli sulla cima del Citerone, dove si svolgerà il sacrificio conclusivo ${ }^{35}$.

\section{Il tema del bagno: miti e procedure rituali}

La descrizione dei Daidala Megala contiene elementi (il bagno, la processione) che legano il nostro passo tanto a quei racconti in cui compare un simulacro come oggetto di operazioni rituali, quanto a specifici momenti dei riti matrimoniali $o$, sarebbe più giusto dire, pre-matrimoniali (come ci sono testimoniati tanto dalla tradizione letteraria quanto dalle fonti iconografiche, in particolare dalle immagini che compaiono sulle loutrophoroi). Gli uni e gli altri trovano un significativo elemento connettivo nella figura di Era.

\section{Kosmesis agalmaton}

19 I testi ci presentano vari esempi in cui un simulacro viene lavato presso un fiume o una fonte, abbigliato, adornato ${ }^{36}$ : si tratta in grande maggioranza di statue di divinità femminili e, tra queste, un posto di rilievo occupa $\mathrm{Era}^{37}$; le fonti ci forniscono d'altra parte conferma del fatto che vesti, stoffe, gioielli (tutto quanto in greco veniva indicato

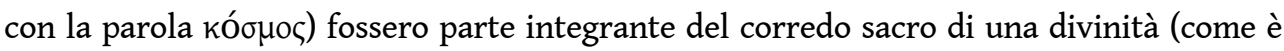
possibile desumere sia dai resti materiali, sia dalle fonti letterarie e gli archivi dei santuari $\left.{ }^{38}\right)$. Come ha mostrato Pirenne-Delforge a partire dal caso di Sicione ${ }^{39}$, le testimonianze di cui disponiamo mostrano il rilievo e l'importanza che tali operazioni dovevano avere, affidate com'erano a 'personale specializzato' ed oggetto, in determinati casi, di particolari privilegi. Riguardo a Sicione, Pausania narra, per esempio, che l'accesso al santuario di Afrodite era consentito unicamente ad una donna in qualità di

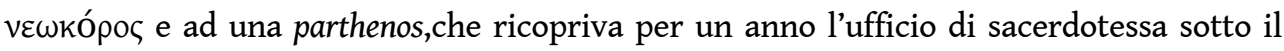
titolo di loutrophoros ${ }^{40}$, termine che "suggère immanquablement l'idée d'un bain" ${ }^{41}$, nonostante le fonti nulla aggiungano in dettaglio, e svela nel riferimento al sacerdozio il carattere sacro che la pratica doveva avere ${ }^{42}$; ancora Pausania riferisce di come, a Olimpia, i discendenti di Fidia, designati come phaidruntai ("lucidatori"), avessero ricevuto quale privilegio ereditario la cura della statua crisoelefantina di Zeus ${ }^{43}$. Ma l'esempio più emblematico di questo genere di pratiche è forse costituito da Plynteria $e$ Kallynteria ateniesi, feste che, già nel nome, rivelano una relazione con le operazioni rituali in questione. Vista la complessità della documentazione ad esse relativa, non è possibile entrare qui nel dettaglio. Mi limito a osservare che, per i Plynteria ${ }^{44}$, tali operazioni sono prerogativa del genos dei Praxiergidai e sono parte di cerimonie segrete ( orgia aporreta)che i membri del genos celebrano in quella occasione ${ }^{45}$. Ulteriori elementi ricaviamo da un'iscrizione ${ }^{46}$ : dal genos dei Praxiergidai erano estratti due loutrides o plyntrides e un kataniptes ${ }^{47}$, informazione che conferma tanto la centralità del 'bagno' nel trattamento rituale del simulacro ${ }^{48}$, quanto il ruolo sacrale, di prestigio, che dovevano avere tali operazioni, al punto da costituire oggetto di prerogative gennetiche ${ }^{49}$. Quanto ai Kallynteria, non possediamo fonti atte a illuminarci sul contenuto della festa, l'unico dato 
che ci lascia intravedere un legame con la pratica della kosmesis lo ricaviamo dalla testimonianza tarda di Fozio, il quale riferisce che toccò per prima ad Aglauro, in qualità

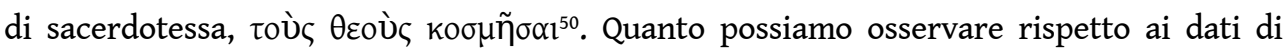
pensiero che la casistica analizzata ci svela, è che l'esigenza - pratica - della manutenzione periodica delle statue costituisce d'altra parte riflesso della concezione stessa del corpo divino che deve, anche nella oggettivazione materiale costituita dal simulacro, sfuggire alle ingiurie del tempo, incarnare bellezza e perfezione ${ }^{51}$.

\section{Bagno e status virginale} declinazioni - elemento di rilievo del nesso mitico-rituale : esso è infatti al centro tanto nelle pratiche di culto relative ad alcune divinità (specie femminili come si è detto), quanto nei racconti mitici in cui le stesse compaiono; nè andrà trascurato che, nella pratica comune dei Greci, il bagno era uno dei momenti essenziali dei riti prematrimoniali ${ }^{52}$, quelli cioè che precedevano immediatamente la processione che attuava il trasferimento della sposa dalla casa paterna a quella dello sposo ${ }^{53}$. Al di là della elementare esigenza purificatoria legata a qualunque contatto con il sacro, è possibile andare oltre nella comprensione del valore attribuito al bagno prenuziale attingendo alle fonti ${ }^{54}$. Il bagno era, a livello generale, una delle pratiche intese a separare l'individuo dal suo status precedente ${ }^{55} \mathrm{e}$ alle acque - particolarmente a quelle dei fiumi - era attribuito un potere fertilizzante, come ci informa uno scolio alle Fenicie di Euripide, proprio in relazione ad un contesto matrimoniale ${ }^{56}$ : dobbiamo intendere dunque che, nel caso delle ragazze, si trattasse della separazione dallo status virginale, come ci è testimoniato per altra via $^{57}$ dalla allocuzione rituale che le fanciulle della Troade rivolgevano allo

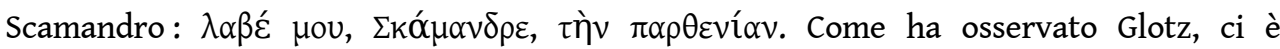
documentata, per la Grecia, l'esistenza di numerosi corsi d'acqua il cui nome era $\Pi \alpha \rho \theta \varepsilon ́ v i o \varsigma$, un dato la cui eziologia sarebbe secondo lo studioso da individuare nella pratica di offrire giovani vergini alle divinità delle acque come dono sacrificale ${ }^{58}$. In tale quadro andrebbe dunque inserita la pratica del bagno prematrimoniale che doveva configurarsi, secondo Glotz, come una prova ordalica attraverso la quale la giovane sposa dava prova della propria verginità al futuro sposo $^{59}$. Personalmente ritengo che tanto l'allocuzione allo Scamandro, quanto la testimonianza offerta dallo scolio alle Fenicie, siano indizi di qualcosa di parzialmente diverso, del fatto cioè - come detto - che il valore del bagno consistesse appunto nella marcatura del momento di passaggio delle giovani dallo status virginale, che bisognava simbolicamente "lavare via", alla nuova condizione cui accedevano con il matrimonio ; nella stessa direzione mi sembra andare la richiamata attestazione, per varie regioni della Grecia, di corsi d'acqua che recavano nel nome un rapporto con le parthenoi.

\section{La processione e l'ambiguo statuto della nymphe}

21 Nel nostro testo, alle operazioni presso l'Asopo segue la $\pi$ rouń, che deve trasportare i 14 simulacri via via realizzati nel corso di ogni celebrazione dei DaidalaMikra fino alla cima

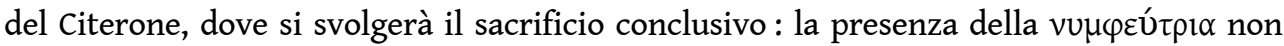
lascia dubbi sul fatto che la rourńdebba essere intesa come corteo nuziale, per fittizio che sia, e sul fatto che, anche nel rito - come già abbiamo visto per l'aition mitico - il daidalon 
è sostituto simbolico di una sposa o, per essere più precisi, di una donna che viene

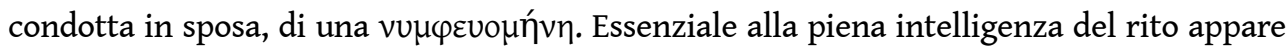
il richiamo di quello che Claude Calame mostra essere lo statuto transitorio della nymphe, non più parthenos, non ancora donna compiuta, gyne $e^{60}$. La logica simbolica sottesa a tale passaggio trova riscontro anche nell'evidenza materiale : non privo di importanza, infatti, appare anche il fatto che statuette di terracotta, che riproducono una figura femminile velata (donna/dea ?) - databili al VI secolo - sono state ritrovate sul sito dell'Heraion di Platea e potrebbero, secondo gli archeologi, essere in relazione con le tipologie statuarie menzionate nel testo di Pausania ${ }^{61}$. Il dato appare tanto più significativo se si considera che il 'disvelamento' della sposa da parte dello sposo era uno degli atti codificati - dal forte valore simbolico come è evidente ${ }^{62}-$ all'interno di una precisa cerimonia, quella degli anakalypteria appunto ${ }^{63}$ : "avant d'acquérir son statut de femme légitimement mariée, la jeune épousée porte un voile. Ce dernier signale un état transitoire : la nymphe a quitté sa famille d'origine et n'est pas encore intégrée à celle de son mari" ${ }^{64}$. É possibile notare peraltro come una conferma a tali dati possa venire dalla lingua, se è vero che l'etimologia, pur oscura, del termine vú $\mu \varphi \eta$, potrebbe secondo l'ipotesi di Chantraine ${ }^{65}$ esser fatta risalire alla radice indoeuropea * ${ }^{*} n e u b h$-, cui è ricollegabile la radice latina nub, che contiene in sè tanto l'idea del nascondere, celare (nubes), quanto quella del matrimonio (nubere, nuptiae). L'anakalypsis in quanto atto che mostrava la donna, fino a quel momento celata, agli occhi dei presenti, veniva ad acquisire dunque anche il valore di sanzione ufficiale della legittimità dell'unione, ulteriormente asseverata dalla dinamica di offerta/accettazione dei doni (anakalypteria), il cui valore obbligante viene a sancire la mutua accettazione del legame matrimoniale alla presenza di testimoni ${ }^{66}$.

\section{Olocausto e divinità olimpie : una conciliazione possibile?}

Quanto del nostro testo rimane da indagare è dunque la possibilità di raccordo tra gli elementi fin qui esaminati e il sacrificio che viene a chiudere, nel racconto di Pausania, la celebrazione dei Daidala Megala.

Ciò che, nella storia degli studi, ha creato maggiori difficoltà a livello interpretativo è infatti costituito dal trattamento finale che i daidala ricevono (l'olocausto ${ }^{67}$ ) e dalla sostenuta incongruenza tra tale trattamento e le forme del sacrificio in onore delle due divinità olimpie: quale cioè il senso di statuette che vengono realizzate per essere distrutte, assieme alle vittime che sono state sacrificate in onore dei due dei, ma delle quali non vengono - come consuetudine vorrebbe - consumate le carni ? e quale la via di conciliazione possibile tra un rito dalle forme così particolari e il fatto che il sacrificio sia offerto a Era e Zeus congiuntamente, come coppia divina ? Richiamando i dati che il testo ci offre in successione e che abbiamo via via esaminato, credo sia utile porre in evidenza che il punto iniziale - l'aition - e il punto finale - il sacrificio (indipendentemente dalle forme che esso assume) - sono perfettamente rispondenti : nell'uno come nell'altro caso a essere in gioco sono Era e Zeus come divinità legate al matrimonio. Congruente con entrambi è il rito intermedio, quello della heorte panbeotica (forma plurale di quella che dobbiamo supporre fosse la festa plateese), che ci mostra il bagno rituale di cui è oggetto

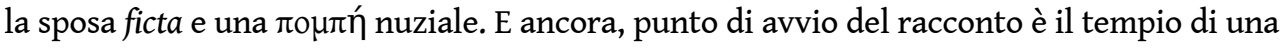
divinità, Era, venerata al suo interno con due epiteti che evocano momento iniziale e momento finale di uno specifico status femminile : quello della donna che viene condotta 
in sposa (Nympheuomene) e quello della donna che ha trovato - con il matrimonio - il suo telos, il suo compimento (Teleia).

Perché, dunque, delle statuette che rappresentano una sposa vengono, a conclusione di un corteo nuziale, bruciate all'interno di un rito i cui destinatari ultimi sono le divinità garanti del matrimonio ? Credo che la spiegazione sia da ricercare proprio nel passaggio dall'una all'altra condizione espresse dai due epiteti cultuali di Era. Il daidalon rappresenta una nympheuomene (si ricordi la esplicitata presenza della nympheutria sul carro ${ }^{68}$ ) : perché essa possa attingere il nuovo status, perché possa entrare sotto la protezione della dea che porterà a compimento il suo telos (credo non sia irrilevante - a riguardo - il valore attivo di Teleia), è necessario che lo status precedente venga cancellato; di qui la particolarità del trattamento rituale riservato al simulacro. Dato problematico rimane invece l'olocausto animale, che non può inserirsi in modo organico in questo quadro, e per il quale andrà pertanto ricercata una diversa spiegazione ${ }^{69}$.

\section{Polivalenza di telos e dimensione matrimoniale}

Quale contesto, a livello umano, dobbiamo dunque immaginare dietro la celebrazione di questi riti nella loro forma più antica $\mathrm{E}$ in che modo è possibile interpretarli nel quadro di una comunità che dà valenza politica a tali riti ? La riflessione che, all'interno della sua storia della religione greca, Louis Gernet dedica alle feste contadine ${ }^{70}$, delinea un quadro che mi pare attagliarsi assai bene alla nostra situazione. Si tratta di un quadro in cui montagne, fiumi e boschi costituiscono altrettanti elementi di un panorama sacro che fa da "scenario" alla pratica dei matrimoni collettivi come parte integrante delle feste contadine stesse, le quali trovano nella celebrazione stagionale della fertilità della natura da parte di demi rurali associati la propria ragion d'essere. È lo stesso scenario in cui - a livello del mito - troviamo ambientate le ierogamie : su montagne come il Citerone, nei Daidala della Beozia (è lo stesso Gernet a citare l'esempio), che vedono la partecipazione di più comunità riunite, accanto ai numerosi altri esempi che il mito ci offre ${ }^{71}$. Si chiarisce allora, per questo tramite, la polivalenza della nozione di telos - iniziazione, compimento, comunione sociale - appartenente a una famiglia di parole che - scrive Gernet - "il est très notable que, dans le vocabulaire religieux, [...] aient spécialement rapport au mariage" ${ }^{72}$, e che trova alla sua base una concezione religiosa dell'unione fra i sessi, cui per il versante femminile - Era presiede con l'epiteto di Teleia appunto ${ }^{73}$. Sulla stessa linea si colloca l'analisi vernantiana dei Daidala come esempio emblematico del carattere funzionale dello xoanon ${ }^{74}$; scrive Vernant: "Ces scénarios de fête concernent moins Hèra comme sujet divin individualisé, que l'épithète de Teleia qui la qualifie : il retient à la fois l'idée du changement d'état que réalise l'union sexuelle, l"accomplissement' du jeune homme et de la jeune femme, intégrés réellement à la société par le mariage [...]. Le xoanon des Daidala [...] permet de mimer [...] cet 'accomplissement' que l'initiation sexuelle apporte au statut de la femme, en même temps qu' au groupe social tout entier et à la nature" 75 . L'inedito vernantiano, benchè interpreti il daidalon - a mio giudizio erroneamente - come un simulacro di Era (in ragione di un supposto pieno rispecchiamento del mito nel rito), coglie tuttavia il valore che credo all'oggetto debba essere dato : quello di 'mimare' un passaggio di status. L'identificazione del daidalon con un simulacro divino, affermata in numerosi contributi, credo sia imputabile a un superficiale appiattimento dei dati che mito e rito distintamente offrono: il rito riproduce elementi di una cerimonia nuziale (pur fittizia) e ciò ha portato a ravvisare in 
esso la messa in scena dello hieros gamos tra Zeus ed Era e a identificare dunque nella statuetta la dea ${ }^{76}$.

\section{La distruzione dei daidala e un 'ciclo mitico' di Era}

In una recente raccolta sulla religion des femmes ${ }^{77}$, Vinciane Pirenne-Delforge e Gabriella Pironti hanno concentrato la propria riflessione sul caso di Era, con particolare riferimento alla relazione tra epiclesi della dea e femminilità ${ }^{78}$. Il percorso critico delle due studiose prende in esame dati che sono di primaria utilità per la nostra riflessione, poichè permettono di sostanziare il percorso che siamo andati descrivendo, mostrando come gli elementi contenuti nel testo di Pausania possano essere pensati come parte di un sistema più ampio ${ }^{79}$. Dietro tale sistema è possibile intravedere un ciclo mitico di Era, nel quale gli aspetti che abbiamo sottoposto ad analisi dovevano configurarsi come altrettante componenti di un quadro organico, del quale a noi rimangono solo elementi apparentemente disconnessi, rappresentati dalle epiclesi e i riferimenti ad alcuni rituali in onore della dea. Partendo dalla testimonianza di Pausania relativa alla trophe di Era a Stinfalo ${ }^{80}$, in cui si racconta di come la dea avesse ricevuto dal suo trophos, Temeno, figlio

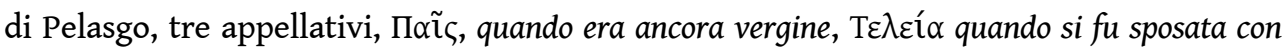
Zeus, Xńpa, quando per una ragione o per l'altra trovatasi in disaccordo con Zeus fece ritorno a Stinfalo, le due studiose mettono in luce come le tre epiclesi individuino tre diversi momenti di uno schema, di cui si trova traccia anche in altre testimonianze relative ad $\mathrm{Era}^{81}$. In particolare, si richiama l'attenzione su un dettaglio che non è stato adeguatamente valorizzato dai contributi critici precedenti, e che è invece a giudizio delle autrici la chiave del process ${ }^{82}$ testimoniato da Pausania. Si tratta della precisazione che Era - a seguito della separazione da Zeus - faccia ritorno a Stinfalo, un particolare fondamentale poichè costituisce la condizione essenziale perchè il processo possa ricominciare : Era è "pais, puis parthenos jusqu' au mariage qui en fait une Teleia" ${ }^{3} \mathrm{e}$ infine, separata da Zeus (Chera), torna al luogo della sua trophe. Quanto Pausania apprende dagli abitanti di Stinfalo è dunque "un cycle mythique transposé dans une liturgie que l'on entrevoit au travers des épiclèses" ${ }^{\prime 4}$. Si deve notare peraltro che si tratta di temi presenti anche anche nel Plutarco tramandatoci da Eusebio: la trophe di Era ancor vergine (in Eubea), il rapimento da parte di Zeus e il relativo nascondiglio in una grotta del Citerone, l'unione segreta tra le due divinità e l'attribuzione ad Era, una volta che l'unione fu scoperta, degli epiteti di Teleia e Gamelios ${ }^{85}$.

Agli elementi del quadro arcadico possono essere immediatamente accostati da una parte il nostro testo, in cui gli epiteti Nympheuomene e Teleia si collocano - come si è detto all'interno di un processo del quale descrivono rispettivamente svolgimento ed esito (cosituito appunto dall'ottenimento dello status di 'sposa perfecta'potremmo dire), e rispetto al quale il ritiro in Eubea può essere assunto quale parallelo con la Chera di Stinfalo ${ }^{86}$; dall'altra parte, la testimonianza del Periegeta relativa al bagno di Era presso la fonte Kanathos a Nauplia, attraverso il quale - secondo gli Argivi - la dea recuperava ogni annola propria verginità. Che tale bagno sia presentato come parte di una $\tau \varepsilon \lambda \varepsilon \tau \eta^{87}$ mi pare ulteriormente supportare la richiamata polivalenza di $\tau \varepsilon ́ \lambda$ o rimarcata da Gernet, e credo mostri in modo forte la necessità di considerare il Teleia che appare nel nostro testo come termine marcato dal punto di vista religioso ${ }^{88}$.

Il legame a lungo cercato tra il daidalon e la figura di Era, che ha condotto vari studiosi a voler vedere nel simulacro una rappresentazione della dea, credo dunque possa passare 
non solo attraverso i dati che mostrano come, nel testo di Pausania, essa compaia quale divinità garante del matrimonio, ma - alla luce delle riflessioni svolte sul ciclo mitico di Era - anche attraverso una presa in considerazione delle differenze qualitative che oppongono la linearità del tempo degli uomini alla circolarità del tempo degli dei. Se, nel caso della dea, è possibile che il processo che trasforma la parthenos in sposa 'perfecta' cominci ogni volta da capo in ragione di uno schema ciclico, che può ripetersi all'infinito, nella realtà degli uomini si tratta "de transformer à chaque fois, d'une génération à l'autre, une parthenos en 'épouse accomplie'. Il s'agit d'intégrer à l'oikos du mari un élément 'exogène' et critique, une nympheuomènè. La prise en charge de ce processus délicat est précisément l'œuvre de la Teleia" ${ }^{\prime 9}$. E a questo processo è direttamente correlata, io credo, la distruzione del daidalon, in quanto procedimento fisico, concreto, in grado di realizzare - per via simbolica - il passaggio da uno status all'altro, in una dimensione caratterizzata dal tempo lineare e che attinge alla ciclicità solo nella iterazione rituale.

Quanto alla possibilità di intendere con certezza 'Teleia' come epiclesi che trova la sua ragion d'essere in una dimensione legata al matrimonio e, più in dettaglio, nella specifica associazione con Era, credo possano richiamarsi ancora alcuni dati. Una ricerca sulla Banca Dati delle Epiclesi Greche $e^{90}$ mostra come si tratti di uno di quei (rari) casi in cui l'epiclesi è esclusiva di una sola divinità ${ }^{11}$, Era appunto, e come, quando accanto a Era Teleia compare come divinità associata Zeus Teleios, anche l'epiclesi del dio sia declinata in senso 'matrimoniale' e non generico. E ancora, una ulteriore testimonianza del Periegeta mi pare fornire un interessante elemento a conferma della coessenzialità di Era rispetto all'istituto del matrimonio ${ }^{92}$ : a quanto ci dice Pausania ${ }^{93}$, ad Afrodite Era le madri compivano secondo consuetudine un sacrificio per la figlia che andava in sposa. Il fatto che 'Era' compaia al posto della epiclesi di una divinità ${ }^{94}$, in un contesto in cui un sacrificio viene compiuto quando una donna va in sposa, mi pare configurare Era come ipostasi stessa del matrimonio, tanto più che l'epiteto è qui associato ad una divinità, Afrodite, tradizionalmente legata all'amore sensuale, quasi che fossero riuniti nel binomio NOME DIVINITÀ + EPICLESI i due versanti che l'unione matrimoniale implica, quello erotico appunto e quello istituzionale ${ }^{95}$.

\section{L'olocausto animale e la dimensione politica}

30 Se l'ambientazione stessa dei Daidala, i tratti che appaiono caratterizzanti tanto a livello dell'aition quanto nella dimensione rituale (nella doppia forma della festa 'minore' e di quella 'maggiore'), sono tutti aspetti che denunciano la sopravvivenza di elementi la cui sostanza rimanda a dati di mentalità assai antichi, come interpretare infine il fatto che, nella forma che ci è offerta dal testo, i Daidala Megala abbiano una chiara valenza politica ? (come dimostrano la menzione del carattere panbeotico, la estrazione a sorte della $\pi \rho о \pi о \mu \pi \varepsilon i ́ \alpha$, la richiesta da parte dei Tebani di prender parte alla festa e l'invio, da parte loro, di offerte come segno di riconciliazione con i Plateesi).

Già la moltiplicazione delle statuette costituisce - come evidente - dato che non si giustifica in relazione all'aition e rappresenta dunque indizio di qualcosa di nuovo, del mutato scenario in cui la cerimonia viene a inserirsi. Quanto mi pare emergere in modo significativo dal racconto di Pausania, pur nell'accumulo di dati, è l'esistenza di una dialettica fra la regione di Platea e Tebe e, ancor più precisamente, l'esistenza di una situazione di contrapposizione rispetto alla quale i Daidala Megala si configurano come 
momento di superamento, come riconciliazione. Il testo è esplicito nel menzionare come la partecipazione dei Tebani alla festa sia il frutto di una riconciliazione coi Plateesi.

È merito di Luisa Prandi aver messo in luce, ancora di recente, come tale contrapposizione tra le due città sia dato che può esser fatto risalire ben al di là dell'epoca per la quale abbiamo la testimonianza del Periegeta ${ }^{96}$. A fronte di una presentazione della Beozia come realtà geopolitica unitaria, che viene retrodatata dalle fonti addirittura all'età eroica ${ }^{97}$, troviamo infatti costante documentazione di episodi di contrapposizione $\mathrm{e}$ scontro tra gli abitanti della regione, episodi spesso originati dalle mire di Tebe in opposizione al ruolo egemonico esercitato dalla Beozia del sud sin da epoca remota ${ }^{98}$. Il momento specifico cui il nostro testo fa riferimento per l'avvenuta riconciliazione fra Tebe e Platea e la richiesta, da parte della prima, di partecipare a una confederazione comune con l'invio di offerte per la festa dei Daidala è il 316 a.C., data in cui la tradizione colloca la rifondazione di Tebe ad opera di Cassandro. Ciò può probabilmente valere per noi quale indicazione del momento in cui una festa, che dobbiamo presumere preesistente in considerazione dell'antichità degli elementi che la compongono ${ }^{99}$, assunse carattere eminentemente politico.

Quanto emerge dal complesso è dunque la necessità di immaginare, alla base della festa panbeotica, il passaggio da una dimensione politica caratterizzata dall'individualismo di singole poleis a una fase in cui la riunione delle stesse all'interno di un organismo politico comune, il koinon, richiede dapprima sanzione ufficiale e poi periodica conferma dei legami istituitition. Conferma di una risemantizzazione della festa viene anche dalle procedure rituali cui il testo, in questa sezione $(3,8)$, fa riferimento.

Uno degli elementi maggiormente problematici rispetto alla organicità di un quadro fortemente incentrato - come si è visto - sugli aspetti matrimoniali è rappresentato dall'olocausto animale; esso rimane elemento 'fuori serie' e costituisce, assieme ad altri tratti già analizzati, forte indizio dello schiacciamento all'interno della narrazione di dati eterogenei e distribuiti lungo una diacronia di cui è difficile cogliere la profondità, ma che ci si svela nelle incongruenze tra singoli elementi. È, a questo livello, necessario distinguere tra le modalità che il sacrificio assume (un olocausto, laddove ci si aspetterebbe piuttosto il consumo della carne delle vittime a suggello dei legami tra le singole comunità) e il contesto generale di una cerimonia avente come destinatari Zeus ed Era : l'attenzione allo scopo cui il rito è inteso può guidare sulla via dell'interpretazione.

In un contributo dedicato ai legami fra tradizione poetica beotica e origine dei Daidala ${ }^{101}$, Maria Rocchi osserva come l'olocausto sia da porre in relazione non con i caratteri degli dei cui il sacrificio è destinato, ma con lo scopo della festa, che si configura come il rinnovamento di una alleanza ${ }^{102}$, e richiama l'attenzione sul dato - ben noto - secondo cui "les Grecs, à l'occasion des serments et des alliances, ne mangeaient aucune partie des victimes immolées" ${ }^{103}$. Alla luce di tali considerazioni, credo sia possibile pensare che la 'fusione', all'interno del testo, di due riti le cui origini devono presumersi distinte (distruzione del daidalon/distruzione delle vittime animali), passi attraverso l'elemento puramente formale del rito, il fatto cioè che si tratti di una eliminazione per il tramite del fuoco. Se la distruzione del $\delta \alpha i ́ \delta \alpha \lambda$ v è funzionale al passaggio di status (parthenos/ nymphe/gyne) che essa simboleggia e realizza, l'olocausto animale si configura come la conservazione - a livello formale - di una specificità rituale motivata, in origine, dalla conclusione di un patto/giuramento, rispetto al quale esso era la forma ordinaria di sacrificio e al cui interno la distruzione dei daidala potè essere risemantizzata come rito di sostituzione. La festa apparirebbe dunque, in tal senso, come iterazione del patto 
originario: "Il est probable qu'au cours des Mégala Daidala, un tel sacrifice caractérisé par l'anéantissement par le feu des victimes animales et des xoana, était offert par les Béotiens qui entendaient renouveler eux-mêmes un pacte : ils voulaient alors renforcer l'engagement commun en reconnaissant volontairement le lien qui les unissait, comme habitants d'une seule région"104. All'interno di tale quadro, la studiosa avanza l'ipotesi che gli xoana potessero divenire allora, ciascuno, figura delle singole comunità partecipanti al rito, le quali venivano in tal modo - attraverso un rito di 'magia simpatica' 105 - a votarsi all'autodistruzione, in caso di rottura del giuramento pronunciato, in modo pressochè identico a quello testimoniato dall'iscrizione cirenaica contenente il $\mathrm{cd}$. 'giuramento dei fondatori' ${ }^{106}$. Si tratta di una ipotesi che mi pare abbia il pregio di fornire una spiegazione, tra l'altro, alla avvenuta moltiplicazione del numero dei daidala, diversamente non motivata rispetto all'aition. A ciò deve aggiungersi che, come fatto rilevare da Carastro, dalle fonti emerge l'importanza del giuramento come "acte fondamental de la vie civique, qui intervient notamment dans le cadre de la réconciliation entre le membres d'une communauté à la suite d'une période troublée" 107.

\section{Matrimonio e 'eukosmia'}

Se è indubbia la conflazione, all'interno di un unico sistema rituale, di elementi che si mostrano differenti per caratterisctiche ed eziologia, bisogna spiegare perchè il rimando alla dimensione del matrimonio persista. La critica infatti ha parzialmente mancato di spiegare per quale ragione tutto ciò avvenga sotto l'egida delle divinità che - coppia divina per eccellenza - si presentano quali garanti del matrimonio, e perchè questo debba presumersi legato a una dimensione sacra che aveva in Era il suo fulcro. In che modo si giustifica la crasi fra rituali dalla chiara valenza matrimoniale e una festa di carattere eminentemente politico? Cosa motiva la giustapposizione della seconda ai primi lungo una linea che viene in qualche modo percepita come di continuità ? L'aspetto nodale credo debba essere individuato nel valore fondante del matrimonio per le istituzioni sociali e lo stabilimento di ogni dinamica di scambio tra le comunità umane ${ }^{108}$.

Nel suo studio dedicato alle metope dell'Heraion di Selinunte ${ }^{109}$, Clemente Marconi mostra come le immagini in esse contenute compongano un ciclo figurativo organico che, inserendosi come elemento significante e dinamico nell'ambito delle feste della dea come l'autore sottolinea ripetutamente - mira a tradurre agli occhi degli 'spettatori' partecipanti al rito l'instaurazione del kosmos, ad opera degli dei, sulla violenza e il disordine : le metope centrali, dominate da un'atmosfera di pace, sono incorniciate da metope laterali che riproducono scene di violenza ${ }^{110}$. Al centro troviamo quel che Pierre Brulé definisce un pantheon genealogico che raffigura, accanto a Crono e Rea, i loro discendenti ; nella metopa centrale, Zeus ed Era : il primo afferra per il polso la dea scalza - la quale, contemporaneamente, scosta il mantello che le vela la testa. "Il complesso di significati veicolati dall'immagine è densissimo e fa tutto perno sui gesti delle figure : la presa al polso e lo svelamento entrano contemporaneamente in causa in vario modo, solo che li si richiami al referente diretto, il rituale matrimoniale, del quale ciascuno separatamente è parte e costituisce un motivo di rilievo" ${ }^{111}$. Sarebbe certo insensato azzardare precise ricostruzioni in assenza di dati di supporto, ma nell'interpretazione del quadro che il testo di Pausania ci offre credo non vada dimenticata l'esistenza, accanto alle due statue di Era, di una statua di $\mathrm{Rea}^{112}$, una presenza che - attraverso il parallelo con il ciclo figurativo selinuntino - non rende 
impossibile ipotizzare il richiamo a una dimensione cosmologica rispetto alla quale il matrimonio fra le due divinità olimpie rappresenta la fondazione di un ordine nuovo, modello, per gli uomini, di una eukosmia - potremmo dire - che si declina anche a livello sociale e politico. Il matrimonio è - si ricordi - già nella tradizione esiodea (accanto al sacrificio e l'agricoltura), elemento di definizione della condizione umana e rappresenta una tappa essenziale nella costituzione dell'universo civico, in quanto istituzione che, nel garantire al cittadino una discendenza legittima, garantisce la riproducibilità della comunità stessa ${ }^{113}$.

A proposito di Selinunte, Marconi scrive : "il matrimonio è alla base dell'intero sistema sociale e non è un caso che le feste della dea siano, anzitutto, feste di rifondazione dopo la dissoluzione; matrimonio e vita della polis sono intimamente legati [...]. Per garantire quest'ultima è necessario salvaguardare l'istituto del matrimonio ed è questo il compito fondamentale di Era"114. Una conclusione che mi pare di poter richiamare anche per il nostro caso, in cui una realtà assai complessa, composta di elementi eterogenei e stratificati, ci si presenta nel riflesso doppiamente mediato di un testo che trova il suo primum movens in immagini, che appaiono a noi quali elementi 'inerti', ma che nella pratica degli antichi acquistavano senso unicamente in quanto componenti essenziali e dinamiche del rito, giacchè lo statuto dell'immagine cultuale è dato dal contesto rituale nel quale la statua è inserita ${ }^{115}$, non esiste di per sé : rappresentazione del dio e rituale per il dio sono indissociabili.

\section{Abbreviazioni}

Corps des dieux: Ch. malamoud, J.-P. Vernant (ed.), Corps des dieux, Paris, 1986 (= Le Temps de la réflexion 7,1986$)$.

DELG: P. CHANTRAINE, Dictionnaire Étymologique de la langue grecque, Paris,1968.

Héra 1997: J. DE LA GENIÈRE (ed.), Héra. Images, espaces, cultes. Actes du Colloque International de Lille (1993), Naples, 1997.

LSCG: F. SOKOLOWSKI, Lois sacrées des cités grecques, Paris, 1969.

Nomima: H. VAN EFFENTERRE, F. RUZÉ (ed.), Nomima. Recueil d'inscriptions politiques et juridiques de l'archaïsme grec, I-II, Rome, 1994-1995.

ThesCRA: AA.VV., Thesaurus Cultus et Rituum Antiquorum, voll. I-III, Los Angeles, 2005.

\section{NOTE}

*. Una prima versione di questo testo è stata discussa presso il Dipartimento di Filologia Classica dell'Università di Pisa nell'ambito del seminario di Letteratura Greca (a.a. 2011-2012), diretto dal prof. Riccardo Di Donato. Ai partecipanti al seminario sono debitrice per varie osservazioni che mi hanno consentito di arricchire il quadro. A Riccardo Di Donato, che mi ha offerto la possibilità di discutere il testo in quella occasione e ne ha seguito le fasi di elaborazione fornendomi suggerimenti preziosi per l'approfondimento dell'analisi, desidero esprimere la mia sincera 
gratidudine. Alessia Dimartino, Mariagiovanna Lauretta, Andrea Taddei hanno con pazienza ed amicizia discusso con me, a varie riprese, il complesso del testo e singole questioni : tutti li ringrazio con riconoscenza e affetto.

1. FGrHist $388 \mathrm{~F} 1$ (= Eusebius, Praep. III, 1,3 e III, 1, 6). Menzione esplicita della festa è contenuta in III, 1, 6; poco prima però (III, 1, 3), Eusebio racconta che, al tempo in cui Era ancor vergine veniva allevata in Eubea, fu rapita da Zeus e trasportata in Beozia, dove si sarebbe unita segretamente al dio, un passo che, pur non fornendo nessun collegamento esplicito con i Daidala, contiene elementi che credo meritino di essere attentamente valutati in relazione alla figura di Era e ad alcuni aspetti della sua vicenda mitica che appaiono legati a quanto - come si vedrà - è possibile evincere dal testo di Pausania.

2. Il testo di Plutarco-Eusebio infatti, pur menzionando esplicitamente l'istituzione dei Daidala a conclusione del litigio fra Era e Zeus, non fornisce alcuna informazione sullo svolgimento della festa. Una utile tavola sinottica, che pone a confronto le due versioni plutarchee (per le quali cf. supra, n. 1) con quella di Pausania, è contenuta in chaniotis (2002), p. 24-25.

3. Complicati computi sono stati proposti per cercare di risolvere l'aporia; si vedano KNOEPFLER (2001) e STRASSER (2004). Un tentativo che voglia cercare una via di soluzione credo debba muovere però dalla incertezza del dato di partenza riferita dallo stesso Pausania relativamente alla cadenza eptaeterica della festa minore. Una soluzione più economica di quelle molto macchinose finora proposte sarebbe allora supporre per i Daidala Mikra un ritmo pentaeterico.

4. Quanto ai Daidala Megala e alla cadenza di sessant'anni riferita dal Periegeta, giusta l'osservazione di Luisa Prandi secondo cui "se l'esilio dei Plateesi ne interruppe le celebrazioni la festa doveva esistere già prima" e dunque "la durata dell'esilio non poteva render ragione delle scadenze di una festa istituita in un momento anteriore ma semmai modificarle de facto" ; PRANDI ( 1983), p. 90.

5. Sui legami che gli oggetti designati come daidala intrattengono con queste due nozioni, cf. FRONTISI-DUCROUX (1975), cap. 4.

6. Per la più recente rassegna bibliografica sui principali contributi dedicati al nostro testo e le relative interpretazioni in essi contenute, si veda CHANIOTIS (2002), cui vanno aggiunti PIRENNEDELFORGE (2008), p. 223-226 ; PIRENNE-DELFORGE - PIRONTI (2009) e PARKER (2011), p. 194-196 e 221222.

7. Che viene definita $\dot{\varepsilon} \circ \rho \tau$ tuttavia.

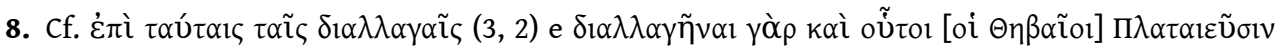

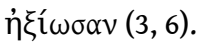

9. CHANIOTIS (2002), p. 24. Lo studioso sottolinea come i Daidala, "with their long and complicated history, point to one of the reason why different approaches in the study of Greek festivals are not only legitimate, but indeed necessary" (l.c., p. 23-24). Sulle modificazioni che la festa deve aver subito, sino ad assumere un carattere eminentemente politico, si veda inoltre CHANIOTIS (2011), p. 264-265.

10. La prima analisi del documento, che fa parte di un gruppo di tavolette tebane provenienti dai ritrovamenti della Odos Pelopidou, è contenuta in GODART - SACCONI (1997), in partic. p. 903-905, ed è stata successivamente edita in ARAVANTINOS - GODART - SACCONI (2001), p. 29-30 e 173-176.

11. La restituzione del termine è resa certa dal confronto con una tavoletta pilia (PY An 292), in cui il vocabolo compare ; cf. GODART - SACCONI (1997), p. 903.

12. Questa l'interpretazione in GODART - SACCONI (1997), p. 904 ; contra BERNABÉ (2008), p. 10-15, in cui si trova un'utile ripresa delle differenti interpretazioni. Bernabé - che riprende esplicitamente la lettura del testo contenuta in MELENA (2001) - contesta in particolare l'interpretazione del composto data dai due studiosi, a partire dalla improbabilità di leggere nella prima parte del termine il riferimento a una divinità, $\Sigma \imath \epsilon \omega ́$, osservazione che appare condivisibile. Più artificiosa appare la lettura, da parte sua, di Te-re-ja-de come $\Sigma \tau \varepsilon l \lambda \varepsilon l o ́$ v $\nu \delta \varepsilon$ in 


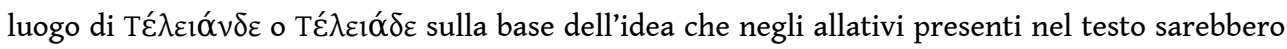
sicuramente da identificare dei toponimi e non altro (p. es. nomi di feste, come vorrebbero Godart e Sacconi ; cf. BERNABÉ, l.c., p. 29 e infra, n. 13).

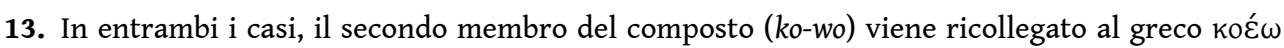
(per il quale si veda $D E L G$, s.v.), sebbene parzialmente divergente sia il significato ad esso attribuito nei due contributi citati: "percevoir, comprendre, entendre, s'occuper de" (Godart Sacconi) vs. "percibir, comprender, entender [...] vigilar" (Bernabé). Si noti peraltro che la serie $A v$ delle tavolette tebane raccoglie specificamente documenti nei quali sono registrati uomini/ donne, talora associati con offerte di grano, cf. GODART - SACCONI (1996), p. 101.

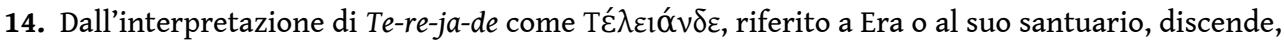
in Godart - Sacconi, la proposta di integrare il]ro-na-de presente alla l.1 della tavoletta in ki-ta-ro$n a-d e$, quale riferimento al monte Citerone come teatro di ambientazione delle feste in onore della dea (l.c., p. 905). Nel commento alla edizione definitiva della tavoletta, gli studiosi scrivono :

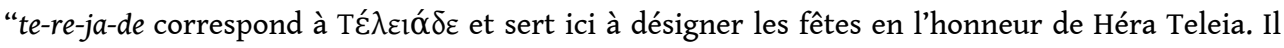
s'agit [...] d'un neutre pluriel suivi du suffixe -de de l'allatif. Ce sont ces fêtes qui, par la suite, deviendront $\tau \grave{\alpha} \Delta \alpha i ́ \delta \alpha \lambda \alpha "$; ARAVANTINOS - GODART - SACCONI (2001), p. 175.

15. Personalmente, se condivisibili mi appaiono le osservazioni di Bernabé circa la difficoltà di ravvisare nel primo membro del composto il nome di una dea ( $\Sigma \imath \omega \hat{)}$ e la conseguente

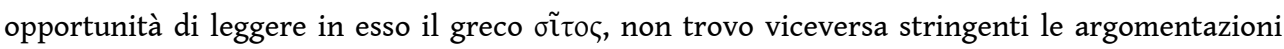
relative agli allativi : dalla esclusione, sulla base di argomenti linguistici (l.c., p. 29), che possa trattarsi del riferimento a nomi di feste, non mi pare discendere infatti la necessità di vedere in essi unicamente dei toponimi, negando recisamente la possibilità che possa trattarsi del riferimento a santuari.

16. SCHACHTER (1981).

17. Si vedano, per quel che ci interessa, SCHACHTER (2000) e (2005).

18. SCHACHTER (2000), p. 13-14.

19. Cf. p. es. Aesch., Eumenides, 213-214, in cui Apollo ribatte al coro delle Erinni (che ha appena

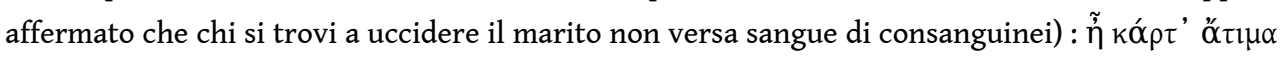

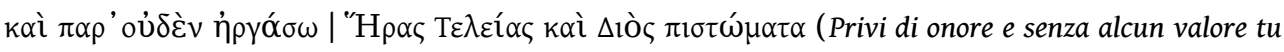
consideri i patti di Era Teleia e Zeus) ; si vedano ancora : Aesch., fr. 383 ; Pind., Nem. X, 18 ; Aristoph., Thesm., 973 ; Diod. Sic., V, 73, 2.

20. Cf. GODART - SACCONI (1996).

21. Gli scavi hanno permesso di identificare l'Heraion più antico in una costruzione, databile al VI sec. a.C., poi distrutta da un incendio (con ogni probabilità durante le guerre persiane), e successivamente ricostruita, secondo quanto apprendiamo da Thuc., III, 68, 3. Relativamente all'epoca delle Guerre Persiane, il racconto erodoteo relativo alla battaglia di Platea (Hdt., IX, 52$53 ; 61 ; 69)$, permette di evincere con chiarezza che il santuario doveva essere il più importante centro di culto della città se, nell'imminenza dello scontro, Pausania pronuncia una preghiera

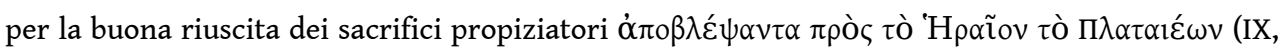
61, 3) ; analogamente, in Plut., Arist., 18, 1, troviamo Era Kithaironia nella lista di dei invocati al momento dell'invasione persiana di Platea. Ad epoca arcaica si datano inoltre alcune statuette fittili, interpretate come dediche ad Era, cf. LIMC, s.v. Hera (in partic. p. 666) e RIELE - RIELE (1966). Per una analisi dettagliata dell'Heraion plateese in relazione ai Daidala, si vedano PRANDI (1983); IVERSEN (2007) che offre un ampio inquadramento storico, dal periodo miceneo all'età ellenistica ; per una prima relazione degli scavi, cf. invece WASHINGTON (1891).

22. A proposito del nome della festa, Vernant indica nei Daidala un caso emblematico, che rende ben evidente come essenziale nel caso degli idoli arcaici fosse non la loro forma, il fatto che riproducessero dei sembianti, ma le operazioni rituali di cui erano oggetto. Nel caso in questione 
infatti la festa prende il nome dall'oggetto stesso che è al centro dei rituali (cf. VERNANT [ds.], p. 57).

23. Il dibattito fra gli studiosi ha oscillato a lungo circa l'opportunità di interpretare le statuette come riproduzioni di un soggetto umano o piuttosto di una figura divina. Il testo in realtà, come si avrà modo di argomentare, non sembra lasciare spazio a dubbi circa il fatto che si tratti di una figura umana e non divina.

24. Chantraine, $D E L G$, s.v. $\delta \alpha 1 \delta \alpha ́ \alpha \lambda \lambda \omega$.

25. FRONTISI-DUCROUX (1975), p. 35. La ricerca è condotta in modo sistematico sulle occorrenze del corpus omerico ed esiodeo e prende a confronto i dati forniti da altri autori.

26. L'analisi della studiosa fa emergere che i termini sono spesso oggetto di verbi che designano azioni tecniche (fare, fabbricare, forgiare) e si ritrovano in associazione significativa con vocaboli che designano utensili e tecniche di lavorazione o che appartengono al campo semantico della luce e del vedere. Essi si trovano inoltre associati con i nomi di alcune divinità ed eroi e hanno spesso relazione con l'ambito della metis e della apate, un legame - quest'ultimo - che si ritrova anche nel nostro testo e che merita di essere approfondito, tanto più che esso è presente anche nel testo plutarcheo ; cf. Eus., Praep. III, 1, 6.

27. FRONTISI-DUCROUX (1975), p. 64-65. Il rimando è a GERNET (1948).

28. Per paralleli, cf. Paus., III, 22, 12 ; IX, 12, 2. L'ultimo passo è relativo al racconto tradizionale sulla fondazione di Tebe, quale leggiamo anche in Eur., Phoen., 638-642, in cui il coro ricorda come il luogo scelto da Cadmo per la nuova città fosse stato, secondo le prescrizioni dell'oracolo delfico, quello in cui lo aveva guidato una giovenca.

29. Si veda p. es. Paus., VIII, 13, 2 e il già citato III, 22, 12.

30. Il dattiloscritto è ora parte delle Archives Jean-Pierre Vernant [AJPV], contenenti le carte dello studioso, ordinate per la cura di Riccardo Di Donato e custodite presso il Laboratorio di Antropologia del Mondo Antico dell'Università di Pisa. Nel regesto, realizzato da Lucia Marrucci, il testo in questione è rubricato in AJPV III.2, fascicolo relativo a La figure des dieux. Essai sur la naissance de l'image chez les Grecs,e occupa le cc. 2-93; per la struttura dell'archivio e la classificazione delle carte al suo interno, cf. DI DONATO (2013a) e MARRUCCI (2013). Le Archives JeanPierre Vernant sono ora interamente consultabili, in formato digitale, sul sito http:// lama.humnet.unipi.it nella sezione 'Archivi'. La sostanza del dattiloscritto vernantiano è stata poi variamente ripresa all'interno dell'opera edita dello studioso e costituisce dichiarata fonte di ispirazione di una parte della riflessione contenuta nella già citata analisi di Frontisi-Ducroux ; cf. FRONTISI-DUCROUX (1975), n. 60, p. 104.

31. VERnANT (ds.), c. 53, corsivi miei. Proprio in relazione al nostro passo, Vernant nota : "Lors des fêtes des Daidala, en Béotie, un rituel compliqué permettait de connaître, chaque année, l'arbre choisi par la déesse pour son xoanon" (ds., c. 54) ; sulla importanza del materiale scelto per realizzare lo xoanon, si veda anche VINCENT (2003), p. 58, che cita, tra vari esempi, anche quello dei daidala.

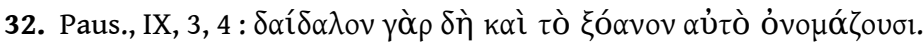

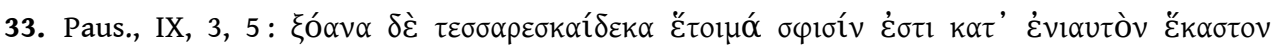

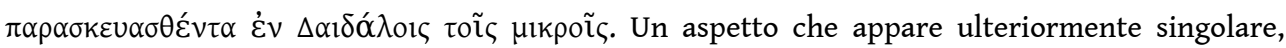
tanto più se si considera il lungo intervallo di tempo che - stando al nostro testimone intercorreva tra le due feste.

34. Su questo aspetto, e sulla possibilità di ipotizzare con buona certezza che i simulacri siano condotti sulle rive dell'Asopo per essere lavati, cf. infra.

35. Paus., IX, 3, 6-7. Dalla descrizione della festa nella sua doppia forma, emerge una sorta di opposizione singolare/plurale, che caratterizza la variante plateese e quella panbeotica rispettivamente, sia per quanto riguarda la dimensione organizzativa e partecipativa (una città 
vs. molte città), sia - conseguentemente - per quanto riguarda l'oggetto stesso della festa (un $\delta \alpha i ́ \delta \alpha \lambda$ ov vs. molti $\delta \alpha i ́ \delta \alpha \lambda \alpha)$.

36. Che tali operazioni dovessero esser parte di una pratica ordinaria ci è d'altra parte testimoniato dalla documentazione epigrafica: alcuni inventari ci forniscono infatti notizie

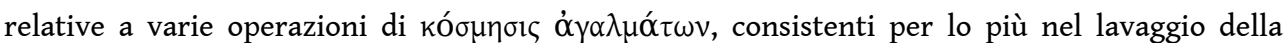
statua e l'unzione con olio; si veda, p. es., IG XI ${ }^{2}, 144,1.37$, in cui è registrata una lista di materiale (una spugna, olio e nitro), destinato alla kosmesis di una statua di Dioniso. Su questi aspetti cf. GINOUVÈs (1962), in partic. p. 283-285. ; KAHIL (1994) e BETTINETTI (2001), p. 143-160.

37. Si vedano, p. es., Paus., II, 38, 2-3 e Athen., XV, 672d, cui va aggiunta la testimonianza relativa alla esistenza, in Argo, di personale sacro specificamente addetto all'acqua del bagno per la dea,

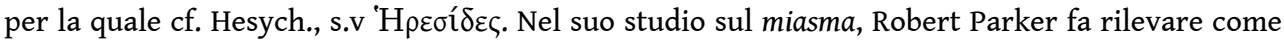
non ci sia ragione di ricercare una spiegazione unica per tutti gli esempi di simulacri divini oggetto di un bagno, data l'eterogeneità dei casi, ma, per quel che ci riguarda, osserva : "The bath that Hera's image receives in Plataea is pre-nuptial"; PARKER (1983), p. 27 ; si noti, per inciso, che Parker - ne deduciamo - considera il daidalon un simulacro di Era. Analoga l'interpretazione del bagno per Christiane Sourvinou-Inwood, la quale a proposito della statua di Era in Argo, scrive : "This loutron was clearly pre-nuptial, as was the bath of Hera's wooden image at the Daidala at Plataia" ; SOURVINOU-INWOOD (2011), p. 188.

38. Che kosmos sia il termine con il quale, tanto nelle fonti letterarie quanto negli archivi dei santuari, viene indicato tutto il corredo sacro che era legato alle singole divinità mi pare deporre in modo significativo a favore della proposta di Matthew Dillon sulla opportunità di non

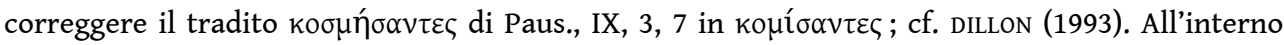

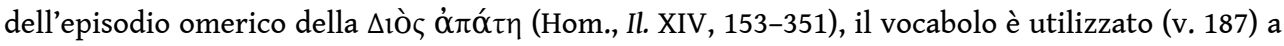
indicare in modo 'riassuntivo' il complesso di ornamenti, precedentemente elencati in dettaglio (vv. 178-186), di cui Era si adorna per sedurre Zeus. Per la documentazione epigrafica si veda, in via esemplificativa, la testimonianza di IG XII, 764, relativa alla Atena di Lindo, in cui è registrato il ricco kosmos di vesti e accessori, di cui la statua viene dotata dagli abitanti della città.

39. PIRENNE-DELFORGE (1994a).

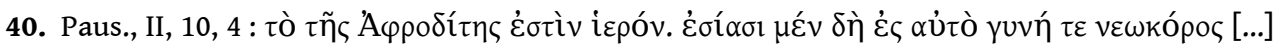

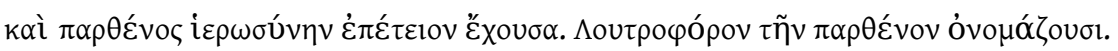

41. Cf. PIRENNE-DELFORGE (1994a), p. 152.

42. Non sappiamo quali forme assumesse il bagno cui l'ufficio di loutrophoros sembra rimandare ; secondo Vinciane Pirenne-Delforge esso è da porre in relazione con l'idea del matrimonio, essendo in causa la dea più direttamente legata all'idea di fecondità ; per questo e per gli altri aspetti legati al bagno della statua e a un inquadramento della figura della loutrophoros, cf. PIRENNE-DELFORGE, l.c.

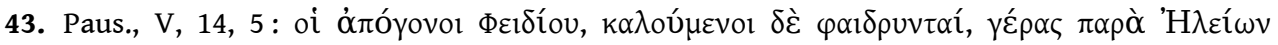

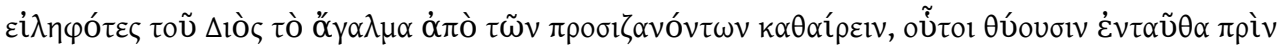

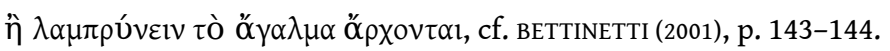

44. Xenoph., Hell. I, 4, 12 ; Plut., Alcib., 34, 1.

45. Plut., Alcib. 34, 1. Per una discussione su questo specifico aspetto, cf. christopoulos (1992).

46. IG I ${ }^{3}, 7$, datata al 460-450 a.C. (= LSCG 15), che riporta un oracolo di Apollo relativo al ruolo e ai patria del genos. L'iscrizione pone varie questioni, assai complesse, che non è possibile affrontare qui in modo approfondito.

47. Per un quadro sintetico su tali aspetti, cf. PARKER (2005), p. 478.

48. Le due feste costituiscono peraltro una conferma del fatto che il bagno delle statue poteva assumere significati diversi a seconda della divinità in questione (cf. supra, n. 37). Qui infatti - a differenza del nostro caso - trattandosi del simulacro di Atena, non è possibile pensare a un bagno nuziale. 
49. Come per il caso già citato dei phaidruntai addetti alla statua di Zeus a Olimpia, per il quale cf. supra, n. 43. Una interessante discussione del decreto relativo ai Praxiergidai è contenuta in LAMBERT (2008), che mette in relazione l'iscrizione con altre relative a doveri rituali legati alle cure della statua di Atena. Lo studioso, sulla base dei dati, arriva a formulare l'ipotesi che tali mansioni rituali, unitamente alle cariche sacerdotali, potessero costituire oggetto di competizione tra gene diversi (cf. l.c., p. 26).

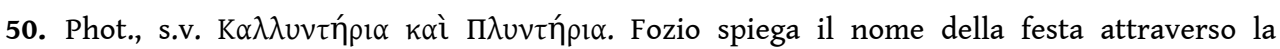

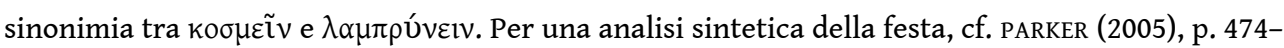
475. Sulla possibile interpretazione di Plynteria e Kallynteria come parti distinte di un unico 'nesso festivo', si veda da ultimo souRvinOU-INWOOD (2011), p. 135-224, con bibliografia precedente ; per gli specifici aspetti che qui ci interessano, cf. invece schMITT-PANTEL (2008), p. 308-311.

51. Alla medesima logica corrispondono tanto le tecniche impiegate (lo sphyrelaton, che prevedeva la copertura parziale o totale del legno con cui la statua era realizzata con un metallo brillante ; la tecnica crisoelefantina che ricorreva all'utilizzo congiunto di oro e avorio), quanto il periodico restauro delle parti più esposte (viso, mani); su tali aspetti, si veda FRONTISI-DUcroux (1986), in partic. p. 260-264. Conferma a tale tipo di concezione viene peraltro dalla

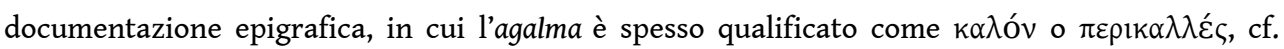
LANÉRÈs (2012), p. 159.

52. Sui valori attribuiti al bagno da parte dei Greci, si veda in generale GINOUVÈs (1962).

53. Per un quadro sintetico su tali aspetti, cf. OAKLEY - SINOS (1993).

54. Pierre Brulé, nel discutere la fase della proteleia, fa rilevare come - al suo interno - il ricorso a una forma di katharsis quale è il bagno trovi spiegazione nella visione del matrimonio come "prova", che richiede al soggetto che vi si sottopone di essere puro, pratica attestata da numerose tradizioni locali, che evocano il momento in cui le giovani donne si recano nei pressi di corsi d'acqua o fonti ; cf. BRULÉ (1987), p. 318-319.

55. GINOUVÈs (1962), passim.

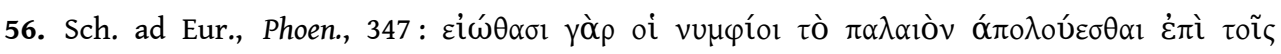

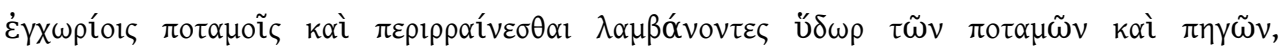

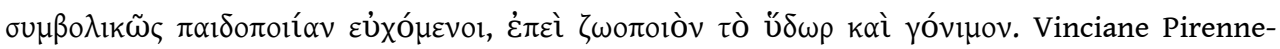
Delforge ha opportunamente sottolineato come quella relativa allo Scamandro sia solo una delle testimonianze che permettono di mostrare come tali virtù fossero attribuite, a seconda delle regioni, a un fiume o una fonte particolari : l'Ismenio a Tebe, lo Scamandro a Troia, la fonte Calliroe ad Atene; cf. PIRENNE-DELforge (1994a), p. 151. A tale potere fertilizzante delle acque sarebbe legato secondo alcuni anche il fatto che i fiumi siano rappresentati spesso nell'iconografia sotto forma di tori, simbolo di virilità.

57. Ps.-Aeschin., Ep. X, 3 ; cf. GERNET (1928) [= GERNET (1968), p. 41-42] ; e GERNET - BOULANGER ( 1932), p. 39.

58. Cf. GLotZ (1904), cap. III ; tra gli esempi citati : Hom., Il. II, 854 ; Hdt., II, 104 ; Xen., Anab. V, 6, 9 e VI, 2, 1 ; Strab., XII, 3, 8. Glotz fa notare come l'Imbraso, il fiume di Samo presso il quale Zeus si unì ad Era e che - secondo la tradizione - era quello in cui ebbe luogo il bagno virginale che precedette il matrimonio sacro, si chiamasse originariamente Parthenios e conclude osservando: "les eaux qui avaient baigné la $\pi \alpha \rho \theta \varepsilon ́$ vo divine en gardèrerent le nom [...] et ne cessèrent jamais d'être associées au culte de l'Hèraion" (o.c., p. 73).

59. O.c., p. 74. Scrive Glotz : "Puisque le dieu de la source voulait des fleurs de virginité, il portait malheur aux jeunes filles qui s'offraient à lui déjà flétries. Il y avait donc là un moyen toujours facile pour une fiancée de montrer à celui qui devait l'épouser qu'elle était digne de lui" (ibidem).

60. CALAME (2009), p. 175.

61. Si vedano, in proposito, SCHACHTER (1981) ; PRANDI (1983) ; PRANDI (1988), p. 23 e LIMC, s.v. Hera. 
62. Simbologia che gioca su un doppio livello: quello, sociale, della 'pubblicizzazione' dell'unione, essenziale a conferire legittimità alla stessa; quello della unione sessuale tra i giovani sposi e del conseguente passaggio della donna dallo status di parthenos a quello di gyne,

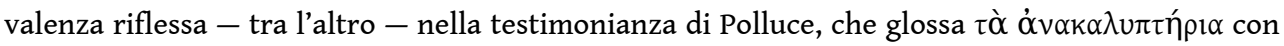

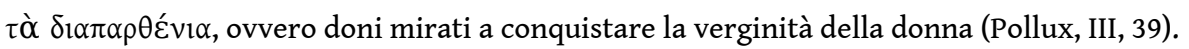

63. Il termine è impiegato dagli autori antichi per designare tanto la cerimonia in sè quanto $i$ doni che, nel corso di questa, lo sposo era costume recasse alla sposa. Quanto alla cerimonia, bisogna tuttavia rilevare che, sulla base delle fonti, non c'è accordo tra gli studiosi circa la fase in cui essa aveva luogo; molti ritengono però assai probabile che si svolgesse nel corso del banchetto di nozze, essendo questo il momento pubblico in cui la comunità era riunita ; sulla questione cf. da ultimo GHERCHANOC (2012), p. 111, con bibliografia precedente.

64. GHERCHANOC (2006), p. 252. Sugli anakalypteria si vedano ancora GERCHANOC (2009), p. 212-217 e GERCHANOC (2012), p.111-113 ; per uno studio incentrato sulla documentazione iconografica, si veda infine DESCHODT (2011), p. 7, la quale insiste sul valore del gesto come espressione dello status della donna sposata e legato, nell'iconografia divina, in modo quasi esclusivo a figure quali Era ed Afrodite. Sulla logica simbolica che presiede al cambiamento, col matrimonio, dello statuto giuridico della giovane sposa, si veda anche CALAME (2009), p. 170.

65. Cf. DELG, s.v. vú $\mu \varphi \eta$.

66. La testimonianza degli oratori mostra con chiarezza il valore attribuito agli anakalypteria i quali, venendo a marcare lo stabilimento di una relazione socialmente identificabile, potevano essere chiamati in causa, nel corso dei processi, nei casi di contestazioni di eredità o cittadinanza; si veda p. es. Is., VI, 64. Sulla peculiarità del matrimonio greco, sancito - a livello giuridico - da una pluralità di atti dal forte valore simbolico, si veda VERNANT (1973).

67. Numerosi sono i contributi che, assumendo come chiave interpretativa proprio l'olocausto, hanno cercato spiegazioni possibili attraverso paralleli con altri contesti in cui il rito compaia. Quella richiamata più di frequente è l'associazione con il culto di Artemide Laphria a Patrai (Paus., VII, 18, 11-13), che presenta tuttavia differenze consistenti rispetto all'olocausto dei Daidala, prima fra tutte il fatto che le vittime vengono gettate nel fuoco non dopo essere state immolate, ma vive; per interpretazioni relative al parallelo con il rito di Patrai,sivedano in via esemplificativa ZUNINO (1994) e, tra i contributi più recenti, PIRENNE-DELFORGE (2008), p. 218-229, e PARKER (2011), p. 167-169.

68. Si noti inoltre che, nella versione plutarchea, è Era stessa ad assumere l'ufficio di nymphagogos, alla testa del corteo formato dalle donne di Platea, mentre la distruzione del daidalon è motivata come atto di gelosia della dea.

69. Considerato il carattere marcatamente politico della festa, ci si aspetterebbe infatti l'usuale spartizione delle vittime a suggello dei legami fra le comunità e fra esse e i loro dei, ma per questo aspetto cf. infra.

70. Cf. GERNET - BOULANGER (1932), cap. I. Riflessione analoga è contenuta, nella forma condensata dell'articolo, in GERNET (1928).

71. Dobbiamo notare che è lo stesso scenario che ci è testimoniato anche da Plutarco.

72. GERNET - BOULANGER (1932), p. 45-46.

73. Su un unico punto, riguardante lo specifico dei Daidala, la ricostruzione gernetiana non mi appare condivisibile: l'interpretazione del daidalon come "fiancée divine" poiché, come si è cercato di argomentare, il testo appare esplicito a questo riguardo.

74. Del fatto cioè che esso acquisti senso unicamente all'interno della dimensione rituale. Si noti inoltre che, se la verifica sull'indice delle CEuvres è corretta, l'esempio relativo alla festa dei Daidala non compare più in alcun luogo dell'opera edita; cf. VERNANT (2007)

75. VERNANT (ds.), p.58-59. Anche in Vernant, come già in Gernet, cui il testo vernantiano si mostra direttamente collegato, il daidalon è interpretato come idolo di Era. 
76. Come già notava Nilsson, e come abbiamo cercato di argomentare, il daidalon è da intendere piuttosto come la riproduzione di una sposa dal punto di vista del rituale; cf. NILSSON (1906), p. 52-53.

77. BODIOU - MEHL (2009).

78. PIRENNE-DELFORGE - PIRONTI (2009), contributo presentato come 'atto primo' di un libro a quattro mani su Era che le due studiose hanno in preparazione ; cf. n. 74, p. 109.

79. Ricostruibile attraverso varie testimonianze delle fonti scritte e del Periegeta in particolare.

80. Paus., VIII, 22, 2-3.

81. Le tre epiclesi scandiscono, secondo Madeleine Jost, uno schema che ricorre nei racconti relativi alle 'dispute fra Era e Zeus', per il quale cf. Jоsт (1997).

82. PIRENNE-DELFORGE - PIRONTI (2009), p. 97.

83. Ibidem.

84. Ibidem.

85. Eus., Praep. III, $1,3$.

86. Su questo aspetto, cf. PIRENNE-DELFORGE - PIRONTI (2009), p. 98.

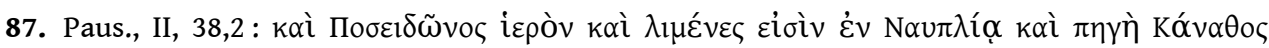

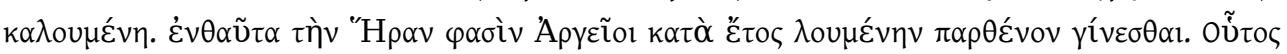

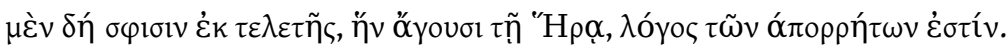

88. Quanto alla localizzazione a Nauplia della cerimonia (il cui carattere annuale permette di pensare a un rituale ciclico), è opinione di Pirenne-Delforge e Pironti che essa possa essere letta come la fase di ritiro della dea dal luogo-principe della sua venerazione, Argo ; cf. l.c., p. 99.

89. PIRENNE-DELFORGE - PIRONTI (2009), p. 109. Per quanto riguarda tale soglia critica, si noti inoltre come un "tratto comune a differenti pratiche che accompagnano il matrimonio" venga individuato da Pauline Schmitt-Pantel nella apate presente anche nel nostro testo, cf. ScHMITTPANTEL (1977), p. 1065 ; la studiosa descrive la apate come l'elemento che compare in numerose ierogamie a rendere possibile l'unione sessuale e analizza diffusamente i legami fra metis e apate e soglia critica rappresentata dal passaggio della donna dallo status di parthenos a quello di nymphe (per questi aspetti si vedano, in partic., p. 1068-1069).

90. Banque de Données des Epiclèses Grecques [BDEG], costruita e messa a punto dal Centre de Recherche et d'Étude des Sociétés et Cultures Antiques de la Méditerranée dell'Università di Rennes 2. La banca-dati è raggiungibile all'indirizzo: http://www.sites.univ-rennes2.fr/lahm/ crescam.

91. Con la sola eccezione di una iscrizione di Delfi, datata al IV sec. a. C., in cui compaiono le

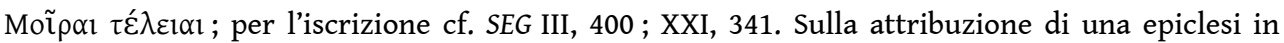
modo esclusivo a una divinità, Pirenne-Delforge e Pironti richiamano il parallelo con Afrodite Ourania ; cf. PIRENNE-DELFORGE - PIRONTI (2009), p. 100.

92. Pirenne-Delforge e Pironti mettono bene in luce come, a differenza di altre divinità femminili, Era non appaia mai dalle fonti come divinità legata alla propria prole o della quale sia enfatizzato l'aspetto materno, ma piuttosto quasi esclusivamente come sposa e sovrana dell'olimpo accanto al marito ; a questa stessa caratterizzazione di Era corrisponderebbe secondo

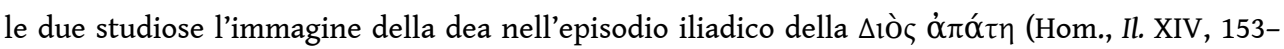
351) ; cf. l.c., p. 104-107, in partic. p. 107.

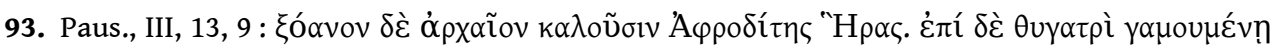

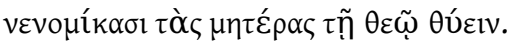

94. Parzialmente diverse le interpretazioni di PARKER 2005a e PIRENNE-DELFORGE 1994 (per la quale cf. infra, n. 95), i quali non ritengono che il secondo nome divino sia interpretabile come una vera e propria epiclesi. Analizzando una serie di casi paralleli in cui compare un doppio nome cultuale (l'associazione cioè dei nomi di due divinità, o di una divinità e un eroe), Robert Parker cita il nostro caso come uno di quelli in cui la formulazione non esprime di fatto l'assimilazione di una 
divinità all'altra, ma "reconnaît plutôt que deux déesses, qui sont d'habitude bien distinctes, dans ce cas précis se rapprochent", una osservazione che mi pare avvalorare l'ipotesi, che qui si propone, della giustapposizione di due aspetti diversi legati alla sfera matrimoniale; PARKER (2005a), p. 225.

95. Come scrive Pirenne-Delforge: "la sphère matrimoniale tout entière trouve dans cette juxtaposition particulière une consécration divine” ; PIRENNE-DELFORGE (1994), p. 198. La studiosa si esprime tuttavia, poco più avanti, circa la possibilità alternativa di interpretare la giustapposizione dei due nomi divini a significare una 'Afrodite Sovrana' (o.c., p. 209-210). Si noti inoltre che ad una Afrodite Era potrebbe rimandare il contesto di un frammento di Alcmane, fr. 126 Calame (= 60 P), per il quale si veda la discussione contenuta in Pirenne-Delforge, o.c. (n. 28), p. 198.

96. Cf. PRANDI (2011). Si veda nel dettaglio il quadro che la studiosa ricostruisce a partire dalle testimonianze di Erodoto e Tucidide (l.c., p. 237-241), che permettono di mostrare come la contrapposizione fra la regione di Platea e Tebe si debba presumere esistente almeno dalla seconda metà del VI sec. a.C.

97. Stante la presenza dell'etnico onnicomprensivo 'Beoti' nel Catalogo delle navi (cf. Hom., Il. II. 494-510).

98. Cf. PRANDI (1988), p. 19-22.

99. Per le argomentazioni a sostegno di tale antichità, cf. supra.

100. Della possibilità di far risalire ad età arcaica il carattere panbeotico della festa è convinta Luisa Prandi, tornata più volte sull'argomento ; cf. PRANDI (1983) ; (1988), e (2011), p. 247-248, in cui è esplicitamente contestata l'ipotesi di Iversen secondo cui l'istituzione dei Daidala Megala sarebbe da connettere con il rientro dei Plateesi dall'esilio del 338 a.C. ; cf. IVERSEN (2007), p. 383384.

101. ROCCHI (1989).

102. Si ricordi come l'istituzione originaria della festa sia esplicitamente motivata come

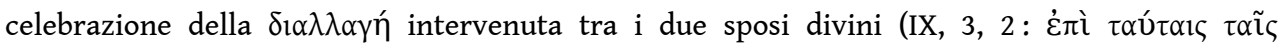

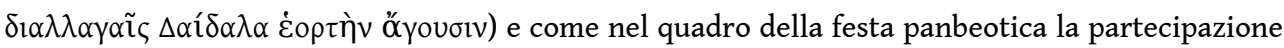

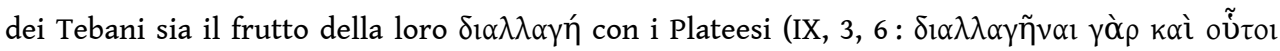

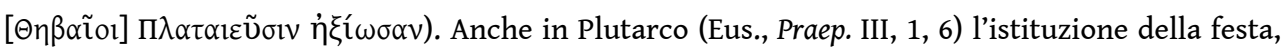
ad opera della stessa Era, è conseguente alla riconciliazione.

103. RocCHI (1989), p. 322. La studiosa cita a supporto Hom., Il. XIX, 266-268. ; Paus., III, 20, 9 ; V, 24 , 10. Sulle forme del sacrificio animale che non prevedevano il consumo delle carni della vittima immolata, si veda da ultimo PARKER (2011), p. 144-150.

104. ROCCHI (1989), p. 323.

105. Cf. FARAONE (1993).

106. Cf. SEG IX, 3 ; MEIGGS-LEWIS (1969), 5 ; Nomima, I, 41. L'iscrizione, contenente il cd. 'Decreto di fondazione di Cirene', sancisce le obbligazioni reciproche intercorrenti tra i coloni che partono alla volta dell'Africa e gli abitanti della madrepatria, Thera ; l'altro parallelo richiamato da Rocchi è quello relativo al giuramento dei re di Atlantide (Plat., Crit., $119 \mathrm{~d}$ ss.). Sulle relazioni tra giuramento e olocausto per i due casi richiamati, si veda GERNET (1951) [= GERNET (1968), p. 207217]. Il caso dell'iscrizione di Cirene è particolarmente vicino a quello dei Daidala poichè il giuramento viene pronunciato gettando nel fuoco dei kolossoi di cera precendentemente plasmati dai contraenti, i quali così, attraverso un rito di sostituzione, invocano la distruzione su di sè e la propria discendenza in caso di spergiuro; cf. GERNET, l.c., p. 213 e VERNANT (1965a) [= VERNANT (1965) [1985'], p. 328-329]. Per argomentazioni e paralleli circa la diffusione nel bacino del Mediterraneo di tali forme di 'magia simpatica' all'interno di rituali di giuramento sin da epoca arcaica, cf. FARAONE (1993). 
107. CARASTRO (2012), p. 89 (corsivo mio). Il contributo oltre a mettere in risalto l'importanza della dimensione sacrificale nei contesti di giuramento, offre una interessante rilettura dei dati dell'iscrizione cirenaica.

108. Per una discussione articolata di questi aspetti in ambito greco, cf. VERNANT (1973).

109. MARCONI (1994). Per il suggerimento, rivelatosi prezioso, di un possibile parallelo con il ciclo figurativo dell'Heraion di Selinunte, sono debitrice ad Alessia Dimartino.

110. Lo stesso schema oppositivo si ripete, analogo, sulle due facciate ; cf. MARCONI (1994), passim, e LÉVÊQUE (1996), p. 95-96. Particolarmente significativo il fatto che nelle metope laterali siano rappresentati personaggi che ricevono la punizione degli dei per essersi in qualche modo posti contro "la buona fisiologia dell'istituzione matrimoniale", cf. MARCONI, o.c., p. 307 ; emblematici in tal senso gli esempi dell'Amazzone, di Atteone, delle Pretidi.

111. MARCONI (1994), p. 277-278. Varie le allusioni simboliche, prima fra tutte il fatto che la dea sia scalza, una condizione che - dai paralleli iconografici - risulta essere simbolo di purezza e dunque, nel caso di Era, richiamo alla sua condizione di parthenos (o.c., p. 287). Si noti peraltro che nel fregio compaiono altre coppie di sposi (Poseidone e Anfitrite ; Ares e Afrodite), divinità non solo "variamente vincolate a Era", ma che "ribadiscono il ruolo dell'istituto matrimoniale nell'assicurare stabilità" (o.c., p. 307). I Daidala sono citati dall'autore come esempio di riplasmazione dello hieros gamos sul matrimonio degli uomini, e le statuette interpretate come simulacri di Era (o.c., p. 281).

112. Paus., IX, $2,7$.

113. Su questi aspetti, con particolare riferimento ad Atene, cf. SCHMITT-PANTEL (1977), p. 10681069.

114. MARCONI (1994), p. 306.

115. A questo riguardo, cf. sCHMITT-PANTEL (2008), p. 307.

\section{RIASSUNTI}

L'article analyse la fête béotienne des Daidala dont Pausanias a conservé le souvenir (IX, 2, 7-3, 8). Tant l'aition (introduit pour expliquer pourquoi l'Héra de Platées porte l'épithète de Nympheomene) que la description de la fête dans sa double forme de Petits et Grands Daidala présentent des aspects qui méritent l'attention. Le texte présente un scénario rituel assez complexe, probablement le fruit d'une longue stratification d'éléments d'origine diverse. La description de la fête que fournit Pausanias pose une série de questions touchant au statut de l'image cultuelle, aux rites pré-matrimoniaux, à l'existence d'un cycle mythique d'Héra lié aux transformations physiologiques de la parthenos, à la signification politique de la fête pour les communautés béotiennes.

This article examines the Boiotian festival of the Daidala as described by Pausanias (IX, 2, 7-3, 8), in its dual form of Small Daidala and Great Daidala. The aetiological myth (aiming to explain why Hera was worshipped at Plataea as Nympheuomene), as well as the description of the festival, are interesting at various levels. The text describes a very complex ritual background, which is the product of a patchwork of elements of different origins. The description provided by Pausanias poses a set of questions concerning the status of the ritual image, wedding rites, the existence of 
a mythical cycle of Hera connected with the physiological transformations of the parthenoi, and the political significance of the festival for the communities of Boiotian worshippers.

\section{AUTORE}

\section{FIORELLA LA GUARDIA}

Laboratorio di Antropologia del Mondo Antico

(http://lama.humnet.unipi.it)

Dipartimento di Filologia, Letteratura e Linguistica (Sez. Greco)

Università di Pisa

fiolag@libero.it 